\title{
Integrator resetting for enforcing constraints in aircraft flight control systems
}

\author{
Kevin McDonough* Ilya Kolmanovsky ${ }^{\dagger}$ \\ The University of Michigan, Ann Arbor, MI, 48109, USA
}

\begin{abstract}
This paper proposes a constraint enforcement scheme based on resetting integrator states in controllers employing integral action. Safe sets of initial conditions for closedloop systems with integral controllers are examined. It is shown that by appropriately choosing the integral states, the response of the system can satisfy constraints for a much larger set of plant initial conditions. A technique based on a solution of a constrained optimization problem is proposed for resetting the integrator state. Simulation results of applying this control scheme to aircraft longitudinal and lateral control are reported. In addition, we consider another application of safe sets for the system with resettable integrator states to plan constraint admissible transitions between aircraft trim points.
\end{abstract}

\section{Nomenclature}

$O_{\infty} \quad$ A safe set with an LQ control scheme

$I_{\infty} \quad$ A safe set with an LQ-I control scheme

$\Delta z \quad$ Deviation of a variable $z$ from nominal value

$U \quad$ longitudinal component of aircraft airspeed in feet per second

$\alpha \quad$ angle of attack in radians

$q \quad$ pitch rate in radians per second

$\theta \quad$ pitch angle in radians

$\beta \quad$ sideslip angle in radians

$p \quad$ roll rate in radians per second

$r \quad$ yaw rate in radians per second

$\phi \quad$ roll angle in radians

$\gamma \quad$ flight path angle in radians

$h \quad$ aircraft altitude in feet

$\delta_{e} \quad$ elevator angle in radians

$\delta_{T} \quad$ throttle position in percent throttle

$\delta_{r} \quad$ rudder angle in radians

$\delta_{a} \quad$ aileron angle in radians

$X \quad$ vector of plant states in nonlinear model

$x \quad$ vector of plant states of linearized model about a specific equilibrium

$x_{I} \quad$ vector of controller states of linearized model about a specific equilibrium

$\bar{x} \quad$ vector of closed-loop plant and controller states of linearized model about a specific equilibrium

\section{Introduction}

Dynamic controllers are frequently used in aircraft flight applications. The states of such controllers can be reset in order to enforce pointwise-in-time state and control constraints. In particular, the integrator state in the basic Linear Quadratic-Integral (LQ-I) control scheme ${ }^{5}$ can be reset to guarantee that constraints are satisfied. In this paper, we apply this integrator reset approach to discrete-time, linear aircraft models for both the longitudinal and lateral dynamics.

\footnotetext{
* Graduate Student Researcher, Department of Aerospace Engineering,

${ }^{\dagger}$ Professor, Department of Aerospace Engineering
} 
For a set of constraints, a safe set is defined as the set of all initial conditions such that the ensuing closed-loop trajectory of the system does not violate the constraints. Such a set is positively-invariant. ${ }^{11,12}$ Previous work on safe sets examined initial conditions of longitudinal and lateral aircraft models in the loop with an LQ feedback controller. ${ }^{7}$ In the current work, we investigate the same models augmented with LQ-I controllers and demonstrate that the set of plant states for which the closed-loop response satisfies the constraints can be enlarged if the possibility of resetting the integrator is taken into account.

We also propose a control scheme for resetting the integrator based on constrained minimization of a Lyapunov function. Our integrator reset scheme is different from the feed-forward command governor described in Kolmanovsky and Sun. ${ }^{3}$ In that work, feed-forward reset is used to improve performance while adhering to constraints. In our case, we reset the integrator states to improve performance and ensure constraint adherence.

Virtual reset absorbers have been proposed in Bupp et $\mathrm{al}^{1}$ (see also Haddad et $\mathrm{al}^{2}$ ). In that work, the closed-loop system energy is reduced through the reset of specific parameters such as the states of a dynamic compensator. However, that work does not consider constraints.

This paper is organized as follows. Section II presents the background and describes the integrator state reset approach. Section III presents the aircraft model and demonstrates the increase in constrained domain of attraction of the closed-loop system if the integrator state can be reset. This section also illustrates the use of safe sets to plan safe transitions between trim conditions of the aircraft model. Section IV ends the paper with concluding remarks.

\section{Problem Statement and Development}

In this section, the closed-loop system model, the definition of a safe set, and the method for integrator state reset are presented.

\section{A. Dynamic System}

Consider the following discrete-time linear system, representing the closed-loop dynamics of a plant with an LQ-I controller,

$$
\begin{array}{r}
x(k+1)=A x(k)+B u(k), \\
x_{I}(k+1)=C_{I} x(k)+x_{I}(k), \\
u(k)=-K x(k)-K_{I} x_{I}(k),
\end{array}
$$

where $x \in \mathbb{R}^{n}, x_{I} \in \mathbb{R}^{m}, u \in \mathbb{R}^{p}$, and $A, B, C_{I}, K$, and $K_{I}$ are matrices of appropriate size. This system can be written in closed-loop form as

$$
\bar{x}(k+1)=\bar{A} \bar{x}(k),
$$

where

$$
\bar{A}=\left[\begin{array}{cc}
A-B K & -B K_{I} \\
C_{I} & I
\end{array}\right],
$$

and

$$
\bar{x}(k)=\left[\begin{array}{c}
x(k) \\
x_{I}(k)
\end{array}\right] .
$$

\section{B. Safe Sets}

A safe set is the set of all initial conditions for (2)-(4) for which the ensuing closed-loop trajectory will not violate constraints imposed on the system. Since (2)-(4) is a closed-loop system, the imposed constraints on the system outputs can reflect either state or control constraints.

The constraints are given by

$$
\bar{y}(k) \in Y_{I}=\{\bar{y}: \bar{G} \bar{y} \leq \bar{f}\},
$$

where $\bar{y}(k)=\bar{C} \bar{x}(k)$ is the output of (2). The safe set is defined as

$$
I_{\infty}=\left\{\bar{x}(0) \in \mathbb{R}^{n+m}: \bar{y}(k)=\bar{C} \bar{A}^{k} \bar{x}(0) \in Y_{I}, \forall k \geq 0\right\} .
$$


Note that $I_{\infty}$ contains safe combinations of the initial conditions for the plant states (the $x(0)$ terms) and for the integrator states (the $x_{I}(0)$ terms). Since constraints (5) are affine, and assuming hereafter that $Y_{I}$ is compact with $0 \in \operatorname{int} Y_{I}, \bar{A}$ is asymptotically stable, and $(\bar{A}, \bar{C})$ is an observable pair, it follows that $I_{\infty}$ is a convex polytope. ${ }^{8}$

\section{Resetting the Integrator State}

Since $x_{I}$ is the controller state, it can be reset to enforce the constraints and simultaneously reduce the closed-loop energy of the system.

A natural selection for a cost function representing closed-loop energy is a Lyapunov function of the form

$$
V(\bar{x}(k))=\bar{x}^{T}(k) \bar{P} \bar{x}(k)
$$

where $\bar{P}=\bar{P}^{\mathrm{T}}>0$ and is the solution to the following discrete-time Lyapunov equation,

$$
\bar{A}^{T} \bar{P} \bar{A}-\bar{P}+Q_{L}=0,
$$

with $Q_{L}=Q_{L}^{\mathrm{T}}>0$.

The value of $x_{I}^{\text {des }}(k)$ for the system (2) is found as a constrained minimizer of (7). That is, at a time instant $k$,

$$
\begin{gathered}
x_{I}^{\mathrm{des}}(k)=\arg \min _{x_{I}(k)} \bar{x}(k)^{T} \bar{P} \bar{x}(k), \\
\text { subject to } \bar{x}(k) \in I_{\infty} .
\end{gathered}
$$

The ability to reset the integrator state online is dependent upon the computing capabilities of the onboard microcontroller to be able to solve the quadratic programming problem (9) online or to store and use its offline-generated explicit solution. The explicit solution can be generated using multi-parametric programming techniques.

When resetting the integrator state according to (9), and assuming the closed-loop system is asymptotically stable, the stability of the origin of the closed-loop system is maintained.

Proposition: Suppose a feasible solution of (9) exists at $k=0$. Then, a feasible solution also exists at all future time instants $k>0$, the constraints (5) are satisfied, and $\bar{x}(k) \rightarrow 0$ as $k \rightarrow \infty$. The origin of the closed-loop system with reset is asymptotically stable with the constrained domain of attraction $\operatorname{Proj}_{x} I_{\infty} \times \mathbb{R}^{m}$.

Proof: Suppose $\bar{x}(k-1) \in I_{\infty}$ is the state at the time instant $(k-1)$ after reset, where $k \geq 1$. Let $\bar{x}^{*}(k)=\bar{A} \bar{x}(k-1)$. By positive invariance properties ${ }^{8}$ of $I_{\infty}, \bar{x}^{*}(k) \in I_{\infty}$ and (9) has a feasible solution, namely $\bar{x}^{*}(k)$, at time instant $k$.

The optimal solution is $\bar{x}(k)$ and, using (7) and (8),

$$
V(\bar{x}(k)) \leq V\left(\bar{x}^{*}(k)\right) \leq V(\bar{x}(k-1))-\bar{x}^{\mathrm{T}}(k-1) Q_{L} \bar{x}(k-1) .
$$

Thus for $N>1$,

$$
\sum_{k=1}^{N} \bar{x}^{\mathrm{T}}(k-1) Q_{L} \bar{x}(k-1) \leq \sum_{k=1}^{N}(V(\bar{x}(k-1))-V(\bar{x}(k))) \leq V(\bar{x}(0)) .
$$

Since $Q_{L}>0, \bar{x}(k) \rightarrow 0$ as $k \rightarrow \infty$. The Lyapunov stability also follows from (10). At $k=0$, $x(0) \in \operatorname{Proj}_{x} I_{\infty}$ implies that a feasible $\bar{x}_{I}(0)$ exists. Hence the domain of attraction is $\operatorname{Proj}_{x} I_{\infty} \times \mathbb{R}^{m}$.

To compare the constrained domains of attraction with and without integrator reset, we define the set

$$
O_{\infty}=I_{\infty} \bigcap\left\{\left(x, x_{I}\right): x_{I}=0\right\}
$$

Clearly, $O_{\infty} \subset I_{\infty}$.

Note that various modifications of the above scheme can be proposed. In particular, the reset can happen at any time instant or at as many time instances as desired after the initial reset. 


\section{Aircraft Examples}

The following aircraft examples are generated using the NASA generic transport model (GTM). ${ }^{13}$ The trim condition for which all of the following results are calculated is, $h_{0}=800 \mathrm{ft}, U_{0}=118.15 \mathrm{fps}$ (70 knots), $\beta_{0}=0 \mathrm{rad}, \gamma_{0}=0 \mathrm{rad}$, and $\dot{\psi}_{0}=0 \mathrm{rad} / \mathrm{s}$. The lateral continuous-time linear model at this trim condition is

$$
\left[\begin{array}{c}
\dot{\Delta} \beta \\
\dot{\Delta p} \\
\dot{\Delta r} \\
\dot{\Delta \phi}
\end{array}\right]=\left[\begin{array}{cccc}
-0.5229 & 0.0861 & -0.9852 & 0.2374 \\
-90.5885 & -6.2736 & 2.0861 & 0 \\
29.1873 & -0.4833 & -1.4043 & 0 \\
0 & 1 & 0.0857 & 0
\end{array}\right]\left[\begin{array}{c}
\Delta \beta \\
\Delta p \\
\Delta r \\
\Delta \phi
\end{array}\right]+\left[\begin{array}{cc}
-0.0002 & 0.0031 \\
-0.9174 & 0.2321 \\
-0.0523 & -0.4436 \\
0 & 0
\end{array}\right]\left[\begin{array}{c}
\Delta \delta_{a} \\
\Delta \delta_{r}
\end{array}\right],
$$

and the longitudinal model is given by

$$
\left[\begin{array}{c}
\dot{\Delta} U \\
\dot{\Delta} \alpha \\
\dot{\Delta q} \\
\dot{\Delta \theta}
\end{array}\right]=\left[\begin{array}{cccc}
-0.0665 & -11.4608 & 0.1439 & -32.1740 \\
-0.0035 & -2.4714 & 0.9514 & 0 \\
-0.0090 & -43.9070 & -3.4738 & 0 \\
0 & 0 & 1 & 0
\end{array}\right]\left[\begin{array}{c}
\Delta U \\
\Delta \alpha \\
\Delta q \\
\Delta \theta
\end{array}\right]+\left[\begin{array}{cc}
-0.0435 & 0.1424 \\
-0.0043 & -0.0001 \\
-0.7662 & 0.0192 \\
0 & 0
\end{array}\right]\left[\begin{array}{l}
\Delta \delta_{e} \\
\Delta \delta_{T}
\end{array}\right] .
$$

Discrete-time models are generated with a time step of $T_{s}=0.1$ seconds. For the lateral model,

$$
C_{I}^{\text {lat }}=\left[\begin{array}{llll}
0 & 0 & 0 & 1
\end{array}\right]
$$

and for the longitudinal model, the integrator is applied to the error in flight path angle, $\gamma=-\alpha+\theta$, thus

$$
C_{I}^{l o n}=\left[\begin{array}{llll}
0 & -1 & 0 & 1
\end{array}\right] .
$$

The weighting matrices in the LQ-I problem were chosen as $Q_{\text {lat }}=\operatorname{diag}\left[\begin{array}{lllll}100 & 100 & 100 & 100 & 1\end{array}\right], R_{\text {lat }}=$ $\operatorname{diag}\left[100\right.$ 100], $Q_{\text {lon }}=\operatorname{diag}[100100100100100]$, and $R_{\text {lon }}=\operatorname{diag}\left[100\right.$ 100]. Also, we chose $Q_{L}=I$ in $(8)$.

The constraints for the lateral linearized system in (5) are

$$
\begin{aligned}
& -0.2618 \leq \Delta \beta \leq 0.2618, \\
& -0.2618 \leq \Delta p \leq 0.2618, \\
& -0.2618 \leq \Delta r \leq 0.2618, \\
& -1.0472 \leq \Delta \phi \leq 1.0472, \\
& -0.5236 \leq \Delta \delta_{a} \leq 0.5236, \\
& -0.5236 \leq \Delta \delta_{r} \leq 0.5236,
\end{aligned}
$$

where $\Delta \beta, \Delta \phi, \Delta \delta_{r}$, and $\Delta \delta_{a}$ are in $\mathrm{rad}$ and $\Delta p$ and $\Delta r$ is in $\frac{\mathrm{rad}}{\mathrm{s}}$. The constraints for the longitudinal linearized system used are

$$
\begin{aligned}
-20 & \leq \Delta U \leq 20, \\
-0.0987 & \leq \Delta \alpha \leq 0.1456, \\
-0.2618 & \leq \Delta q \leq 0.2618, \\
-0.6981 & \leq \Delta \theta \leq 0.6981, \\
-0.5236 & \leq \Delta \delta_{e} \leq 0.5236, \\
-20 & \leq \Delta \delta_{T} \leq 20,
\end{aligned}
$$

where $\Delta U$ is in $\frac{f t}{s}, \Delta \alpha, \Delta \theta$ and $\Delta \delta_{e}$ are in $\mathrm{rad}, \Delta q$ is in $\frac{\mathrm{rad}}{\mathrm{s}}$, and $\Delta \delta_{T}$ is in percent throttle.

The constraints (16)-(17) are intended to reflect both actual plant and controller state constraints as well as constraints which ensure that the linearized model remains accurate. Note that these constraints are specific to the chosen trim condition. 


\section{A. Resetting the integrator state initially to ensure state membership within $I_{\infty}$}

The results presented in this section demonstrate that through resetting the integrator state, constraint admissible transitions from a larger set of plant states are possible, i.e., the constrained domain of attraction is enlarged. The initial conditions were originally selected as $\bar{x}(0)_{\text {lat }}=[0,0.15,0.2,0.50,0]^{T}$ and $\bar{x}(0)_{\text {lon }}=$ $[4,0.05,0.1,0.05,0]^{T}$ with both the lateral and longitudinal integrator states set to 0 . The method described in section II is used to calculate new values for the integrator state at $k=0$ as $x_{I \text { lat }}=-11.9959$ and $x_{I_{\mathrm{lon}}}=5.0534$. The integrator state is only reset initially in the simulations featured in this subsection. Because $O_{\infty}$ and $I_{\infty}$ for these examples are, respectively, four and five dimensional polytopes, here and thereafter projections of these polytopes onto two dimensions are used to convey the relevant information.

The results are reported in Figures 1-3. In Figure 1, a projection of $I_{\infty}$ is displayed in blue and a projection of $O_{\infty}$ is displayed in purple. The green dashed line shows the closed-loop state trajectory of the system with $x_{I}(0)=0$ for the lateral dynamics while the solid red line shows the trajectory with the reset initial integrator state. Note the trajectory exiting the projection of $I_{\infty}$ is indicative of violation of at least one of the constraints.

Note that $I_{\infty}$ is substantially larger than $O_{\infty}$. Note also that by resetting the integrator state, the trajectory does not violate the constraints unlike the trajectory with the integral state set to zero. By resetting the integrator state, a previously unsafe closed-loop response is now made safe.

Figures 2 and 3 show the trajectories of both the lateral and longitudinal closed-loop systems, respectively, projected onto the planes of each plant state with the respective integrator state. In these Figures, $I_{\infty}$ is in blue. Note that without the initial integrator state reset, the trajectory can exit $I_{\infty}$, indicating violation of at least one of the constraints.

\section{B. Repeatedly resetting the integrator state}

The previous subsection described the benefit of resetting the integrator state at time instant $k=0$. This section demonstrates the benefit of resetting the integrator at each time step. The time step used in the simulations is $T_{s}=0.1 \mathrm{sec}$ and the initial conditions at $k=0$ are the same as those used in the previous section. Figures 4 and 5 show examples of the trajectories relative to $O_{\infty}$ and $I_{\infty}$ similar to what was presented in Figure 1, however, the solid red line represents the trajectory with the repeatedly reset integrator state where as the dashed green line represents the trajectory with the integrator state only initially reset.

Figures 6 and 7 are similar to Figures 2 and 3 in that they show the difference between the two methods with respect to projections of $I_{\infty}$ on the plant and integrator states. Note that for each plot, the trajectory resulting from repeated resets of the integral state produces a shorter path through the projected space.

Figures 8-11 show the state and control convergence, excluding the integrator state, for the different trajectories. Note that in each of these four figures, by resetting the integrator state at each time step, the states and control inputs converge more quickly to the origin. Note that the initial condition used for these simulations is an element of $I_{\infty}$ but not an element of $O_{\infty}$.

\section{Constraint admissible flight planning via $I_{\infty}$}

In this subsection, we demonstrate that the safe sets, $I_{\infty}$, can be exploited for constraint admissible flight planning. Towards this end, we consider their application to a longitudinal aircraft dynamics model. Note that a longitudinal trim condition is uniquely defined by prescribing an airspeed, $U_{0}$, flight path angle, $\gamma_{0}$, and altitude, $h_{0}$. The basic approach is similar to references $[7,9,10]$, however, distinguished by the use of $I_{\infty}$ sets in delineating constraint admissible transitions.

Let $X$ denote the absolute aircraft plant state and let $X_{e q}^{i}$ represent a set of trim points (the so called virtual net) that can be commanded to the aircraft fight control system. The two trim points $X_{e q}^{i-}$ and $X_{e q}^{i+}$ are connected if $X_{e q}^{i-}-X_{e q}^{i+} \in \operatorname{int} \operatorname{Proj}_{x} I_{\infty}^{i+}$. The constrained aircraft flight planning problem then reduces to a graph search to identify a sequence of connected trim points leading from the initial trim state to the final trim state.

The execution of the flight path proceeds as follows. Let $i(k) \in\{1, \cdots N\}$ designate the index of the currently commanded trim point. Suppose the operation is currently with the trim point index, $i(k)=i^{-}$, 
i.e., the commanded trim point is $X_{e q}^{i-}$. At a time instant, $k$, we check if the condition

$$
\left[\begin{array}{c}
X(k)-X_{e q}^{i+} \\
x_{I}(k)
\end{array}\right] \in I_{\infty}^{i+},
$$

where $i^{+}=\min \left\{i^{-}+1, N\right\}$, can be satisfied by a selection of the integrator state, $x_{I}(k) \in \mathbb{R}^{m}$. If a feasible solution exists, then the switch $i(k)=i^{+}$is made, resulting in an update to the current trim condition and feedback control gains, while the integrator state is appropriately reset to the feasible value thereby found. We note that $I_{\infty}$ sets used in the above procedure are based on local (linearized) models at various trim points. Approaches to account for the effects of nonlinearities will be considered in the future work.

The following example illustrates this procedure. The desired transition is from an initial trim condition of $h_{0}^{0}=800 \mathrm{ft}, U_{0}^{0}=151.90 \mathrm{fps}(90 \mathrm{knots})$, and $\gamma_{0}^{0}=0.0873 \mathrm{rad}(5 \mathrm{deg})$ to $h_{0}^{N}=800 \mathrm{ft}, U_{0}^{N}=151.90 \mathrm{fps}(90$ knots), and $\gamma_{0}^{N}=-0.0873 \mathrm{rad}(-5 \mathrm{deg})$. This transition is not allowed initially due to imposed constraints. However, if intermediate trim points are added with $\gamma_{0}^{1}=0.0698 \mathrm{rad}(4 \mathrm{deg}), \gamma_{0}^{2}=-0.0524 \mathrm{rad}(3 \mathrm{deg})$, $\gamma_{0}^{3}=0.0349 \mathrm{rad}(2 \mathrm{deg}), \gamma_{0}^{4}=0.0175 \mathrm{rad}(1 \mathrm{deg}), \gamma_{0}^{5}=0 \mathrm{rad}(0 \mathrm{deg}), \gamma_{0}^{6}=-0.0175 \mathrm{rad}, \gamma_{0}^{7}=-0.0349 \mathrm{rad}$, $\gamma_{0}^{8}=-0.0524 \mathrm{rad}$, and $\gamma_{0}^{9}=-0.0698 \mathrm{rad}$, thus $N=10$, this allows for the desired transition to occur with a constraint admissible trajectory.

Figures 12 and 13 show the state and control trajectories of this transition. Note that these Figures plot absolute values of the states and the control. The red vertical dashed lines show at what time instants the switch has occurred to a new trim condition. The first switch occurs at $k=0$ as the aircraft begins its transition from its initial trim of $\gamma_{0}^{0}=5 \mathrm{deg}$ to the next trim of $\gamma_{0}^{1}=4 \mathrm{deg}$. Note that in these Figures, the integrator state is reset at each time instant and this accounts for the vertical jumps in the integrator state plot in Figure 13.

\section{Conclusions}

The states of the dynamic controllers can be reset to increase the constrained domain of attraction for the closed-loop system. While a single reset at the initial time only is sufficient to ensure constraints are satisfied, repeated reset to minimize a measure of closed-loop energy given by a Lyapunov function can also improve the speed of response while maintaining the closed-loop stability. In this paper, these ideas were illustrated in the case of LQ-I controllers with integral action for the control of lateral and longitudinal aircraft dynamics.

In principle, integrator reset techniques can be incorporated into other control architectures; the LQ-I architecture was selected because it is well-established and frequently used in flight control applications.

\section{Acknowledgments}

This work has been supported by NASA Cooperative Agreement NNX12AM54A and by the National Science Foundation Award Number 1130160. The authors would like to thank Professor Ella Atkins and Professor Dennis Bernstein for the collaboration on loss of control of aircraft prevention and recovery that motivated this research.

\section{References}

\footnotetext{
${ }^{1}$ Bupp, R. T., Bernstein, D. S., Chellaboina, V. S., and Haddad, W. M., "Resetting virtual absorbers for vibration control", Journal of Vibration and Control, 6(1), 61-83.

${ }^{2}$ Haddad, W.M., Chellabonia, V., and Neresov, S.G., Impulsive and Hybrid Dynamical Systems: Stability, Dissipativity, and Control, Princeton University Press, 2006.

${ }^{3}$ Kolmanovsky, I. V., and Sun, J., "Parameter governors for discrete-time nonlinear systems with pointwise-in-time state and control constraints", Automatica, 42(5), 841-848.

${ }^{4}$ Lombaerts, T., Chu, Q., Mulder, J., and Joosten, D. "Flight control reconfiguration based on a modular approach", In Fault Detection, Supervision and Safety of Technical Processes, 259-264. 2009.

${ }^{5}$ Wise, K. A., "Affordable flight control for aircraft and missiles," in Perspectives in control engineering: technologies, applications, and new directions, edited by T. Samad, Wiley, 259-290, 2000.

${ }^{6}$ Bayen, A.M., Mitchell, I.M., Osihi, M.K., Tomlin, C.J., "Aircraft autolander safety analysis through optimal control-based reach set computation", Journal of Guidance, Control, and Dynamics, 30(1), 68-77.

${ }^{7}$ McDonough, K., Kolmanovsky, I., Atkins, E., "Recoverable sets of initial conditions and their use for aircraft flight
} 
planning after a loss of control event", Proceedings of 2014 AIAA Guidance, Navigation, and Control Conference, Baltimore, $\mathrm{MD}, 2014$.

${ }^{8}$ Gilbert, E.G., and Tan, K.-T., "Linear systems with state and control constraints: The theory and application of maximum output admissible sets", IEEE Transactions on Automatic Control, 36(9), 1008-1020, 1991.

${ }^{9}$ Weiss, A., Baldwin, M., Erwin, R.S., Kolmanovsky, I., "Spacecraft Constrained Maneuver Planning Using Positively Invariant Constraint Admissible Sets", In Proceedings of Itzhack Y. Bar-Itzhack Memorial Symposium on Estimation, Navigation, and Spacecraft Control, 459-474, Haifa, Israel, 2012.

${ }^{10}$ Weiss, A., Petersen, C., Baldwin, M., Erwin, R.S., and Kolmanovsky, I.V., "Safe Positively Invariant Sets for Spacecraft Obstacle Avoidance," AIAA Journal of Guidance, Control, and Dynamics, 1-13, 2014.

${ }^{11}$ Kolmanovsky, I., Gilbert, E.G, "Theory and computations of disturbance invariant sets for discrete-time linear systems", Mathematical Problems in Engineering, 4(4), 317-367, 1998.

${ }^{12}$ Blanchini, F., and Miani, S., Set-Theoretic Methods in Control, Birkhauser, Boston, MA.

${ }^{13}$ Cunningham, K., Foster, J., Morelli, E., and Murch, A., "Practical application of a subscale transport aircraft for flight research in control upset and failure conditions", 2008, http://ntrs.nasa.gov/archive/nasa/casi.ntrs.nasa.gov/20080034480. pdf.

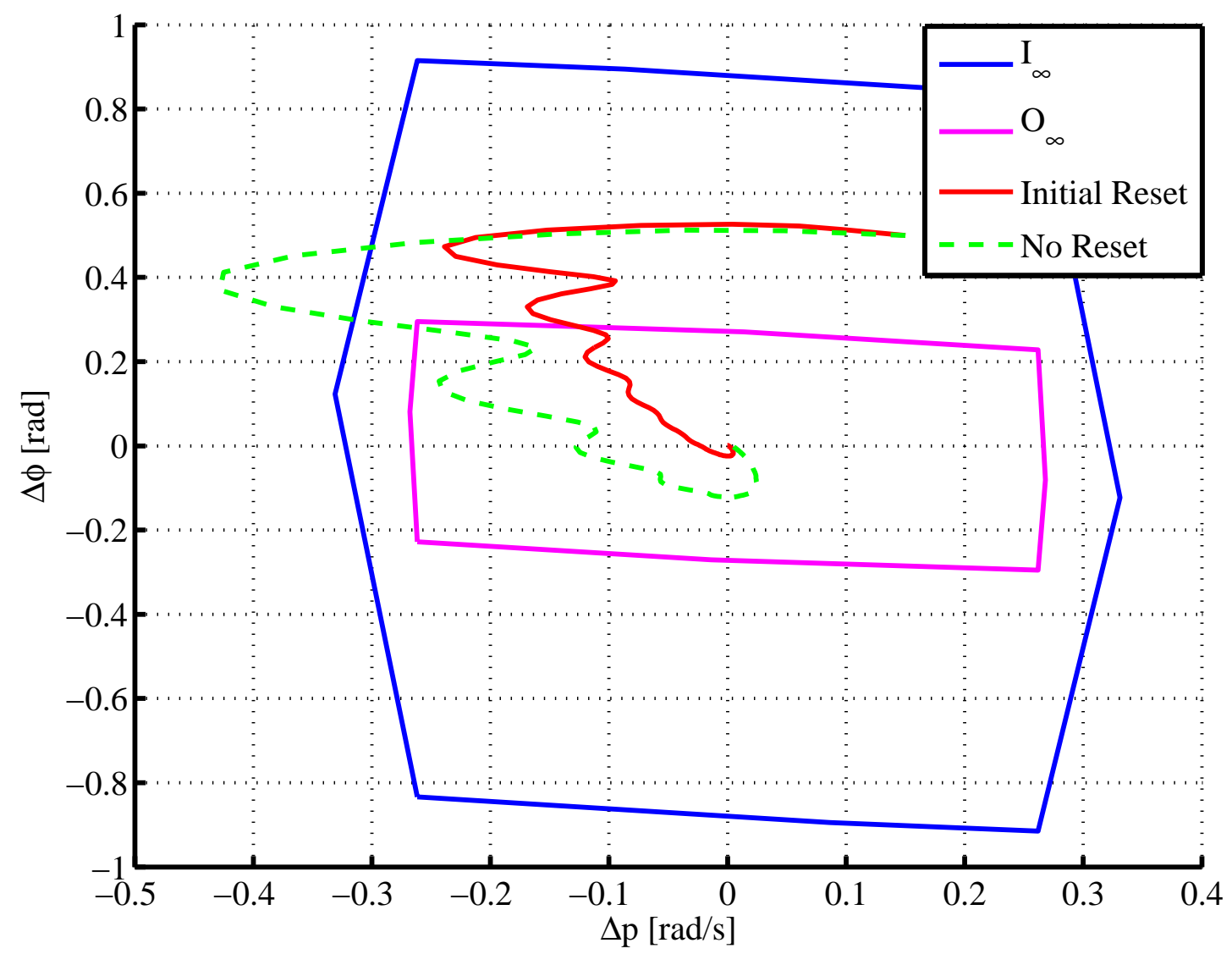

Figure 1: Projections of $O_{\infty}$ and $I_{\infty}$ onto the $\Delta p-\Delta \phi$ plane and closed-loop trajectory of lateral dynamics with and without integrator reset. Note that resetting the integrator state results in a trajectory that does not violate constraints (which are projections of bounds of $I_{\infty}$ in this example). Also note that the projected area of $I_{\infty}$ is substantially larger than $O_{\infty}$. 

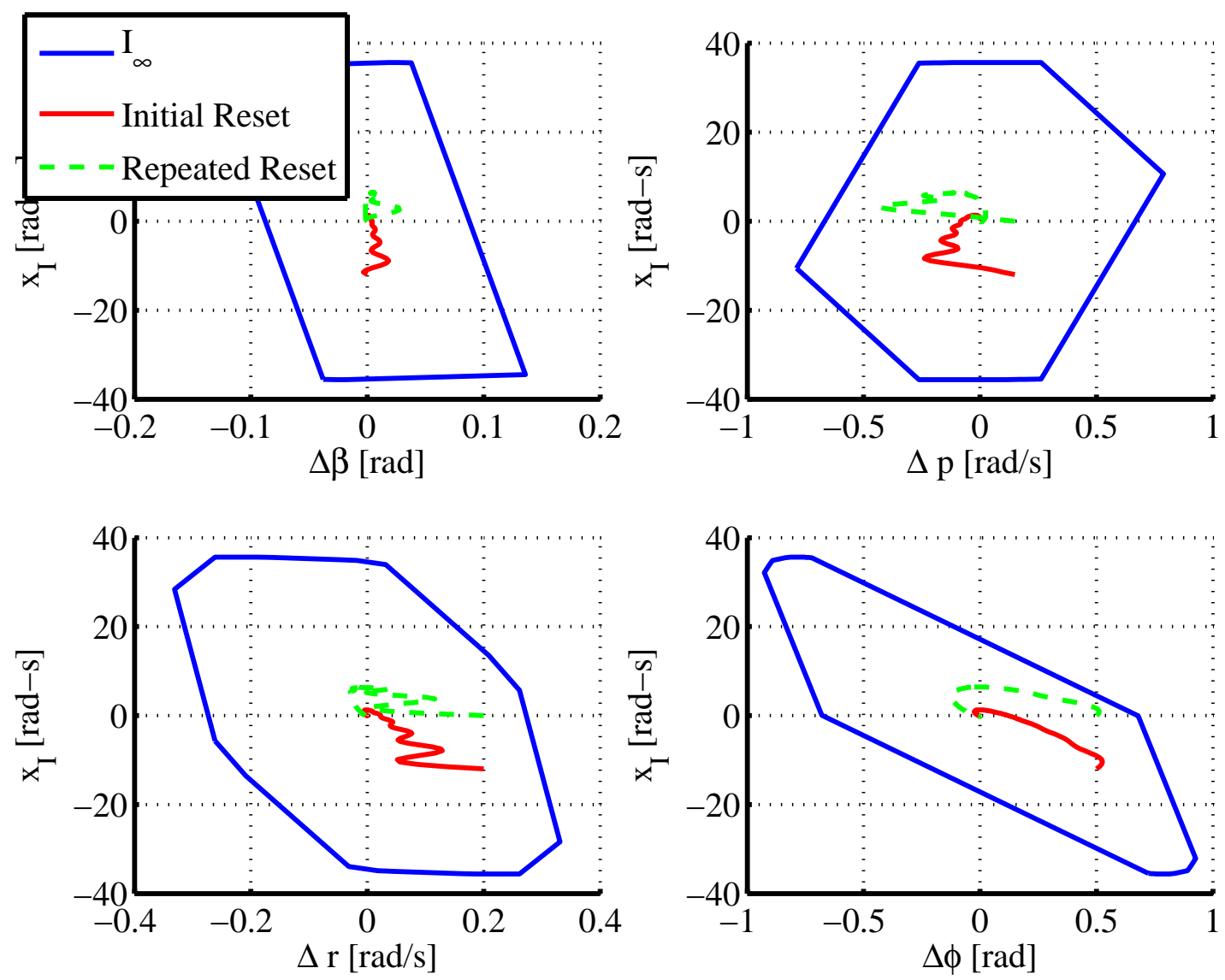

Figure 2: Projections of $I_{\infty}$ onto different planes and closed-loop trajectories of the lateral dynamics with and without integrator state reset. 

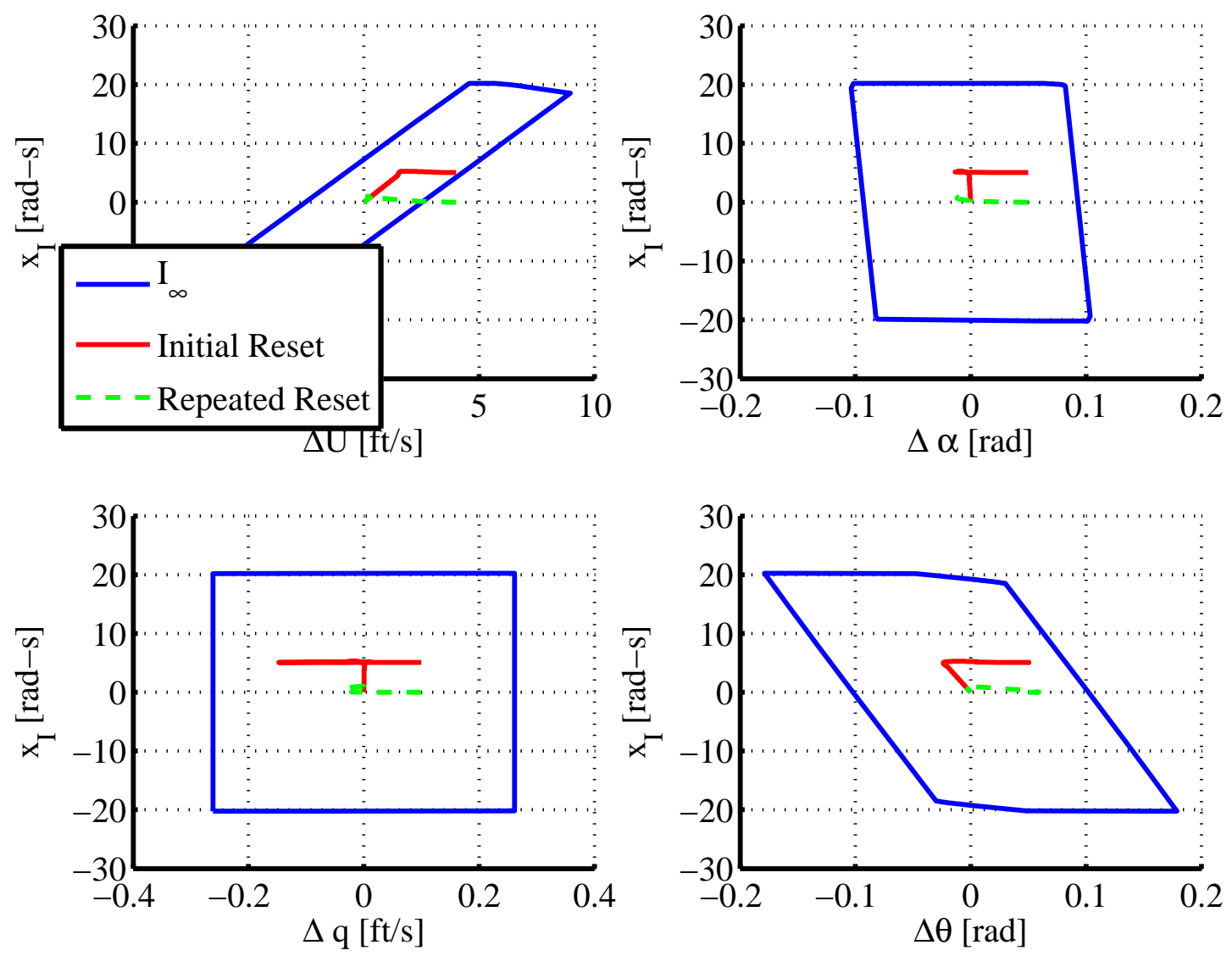

Figure 3: Projections of $I_{\infty}$ onto different planes and closed-loop trajectories of the longitudinal dynamics with and without integrator state reset. 


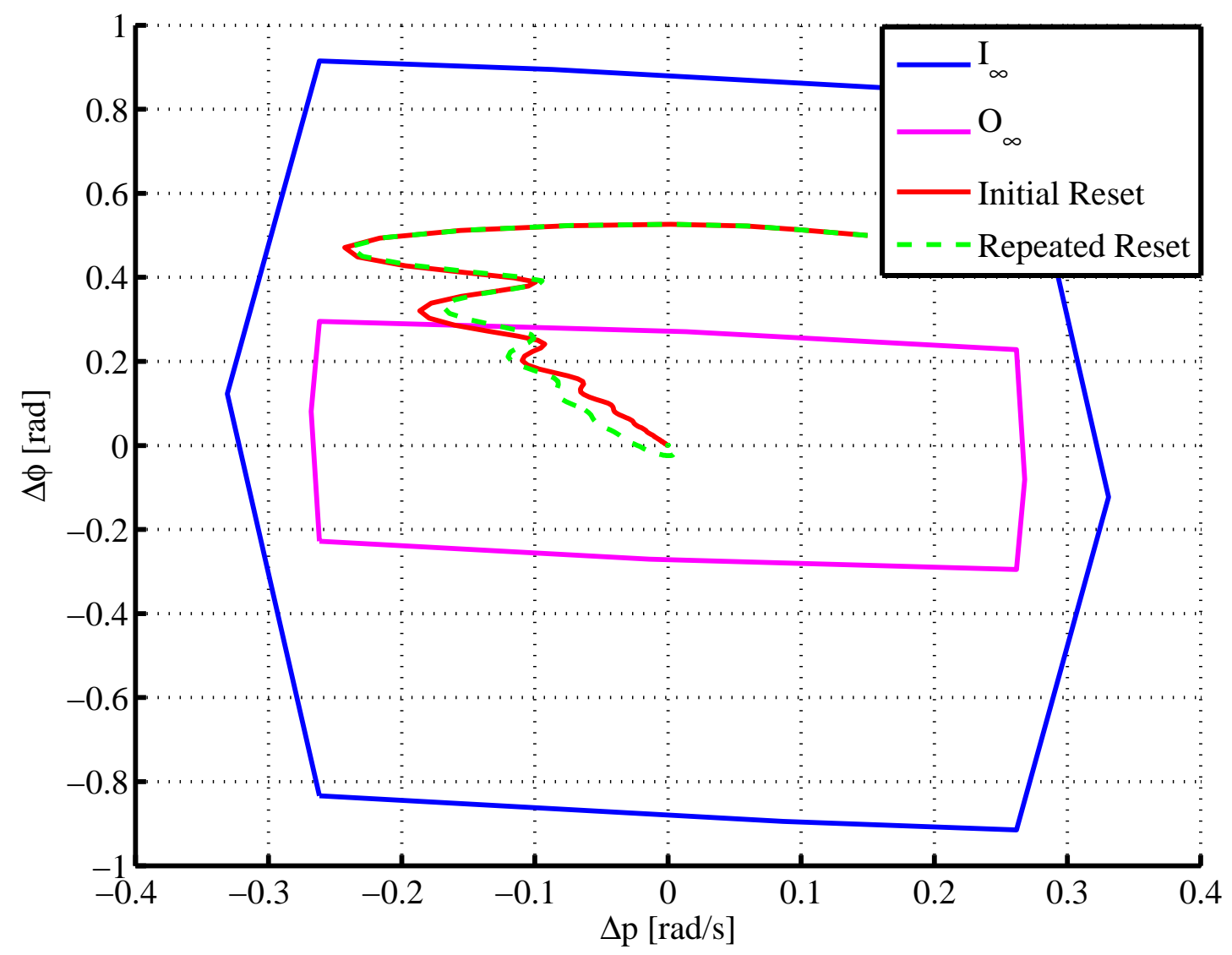

Figure 4: Projections of $O_{\infty}$ and $I_{\infty}$ onto $\Delta p$ - $\Delta \phi$ plane and closed-loop trajectory of the lateral dynamics with an initial reset of the integrator state and with repeatedly resetting the integrator state. Note that repeatedly resetting the integrator state results in a shorter trajectory. Also note that the projected area of $I_{\infty}$ is substantially larger than that of $O_{\infty}$. 


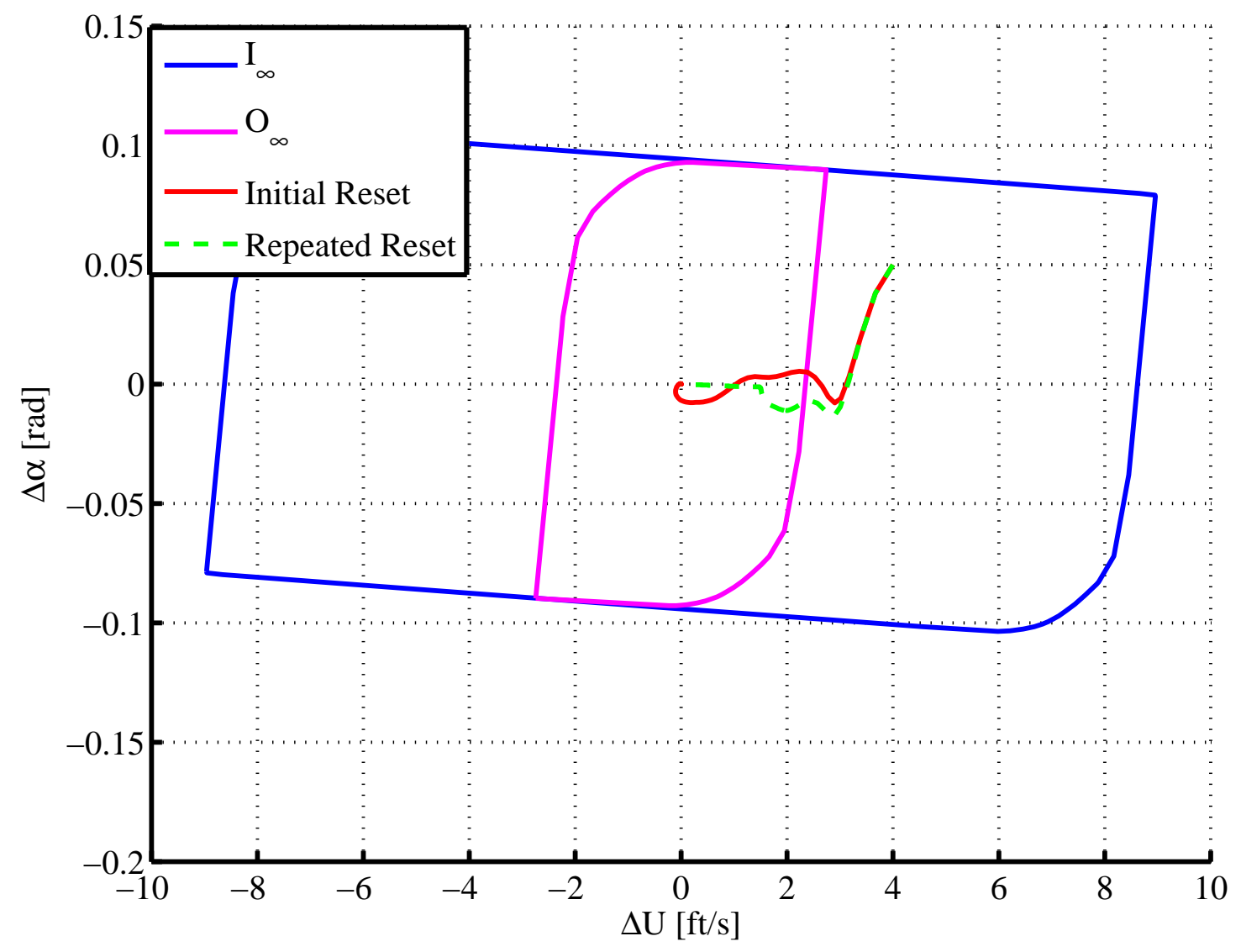

Figure 5: Projections of $O_{\infty}$ and $I_{\infty}$ onto $\Delta u-\Delta \alpha$ plane and closed-loop trajectory of the longitudinal dynamics with an initial reset of the integrator state and with repeatedly reseting the integrator state. Note that repeated resetting the integrator state results in a shorter trajectory. Also note that the projected area of $I_{\infty}$ is substantially larger than that of $O_{\infty}$. 

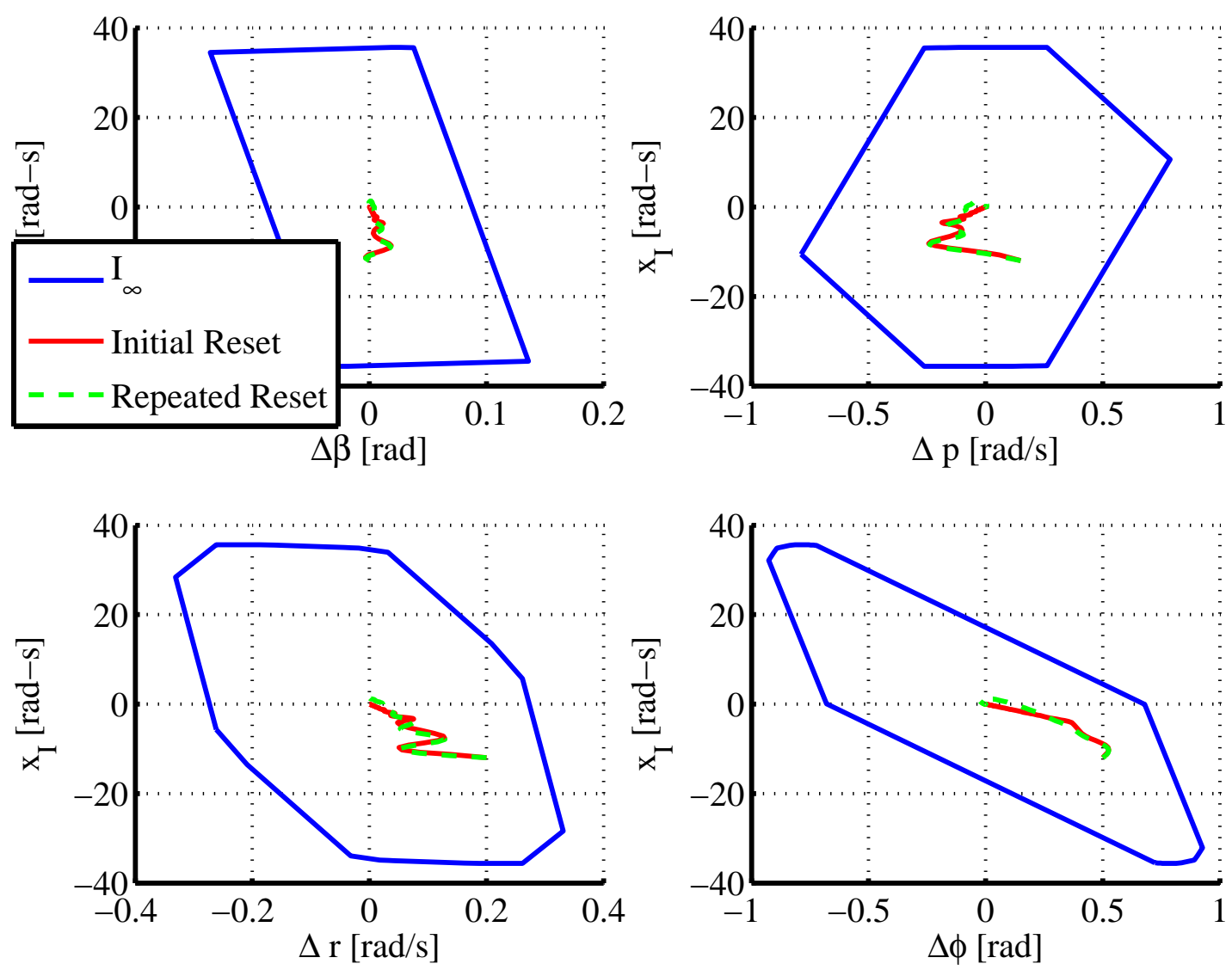

Figure 6: Projections of $I_{\infty}$ onto different planes and closed-loop trajectories of the lateral dynamics with an initial integrator state reset and with repeatedly resetting the integrator state. 

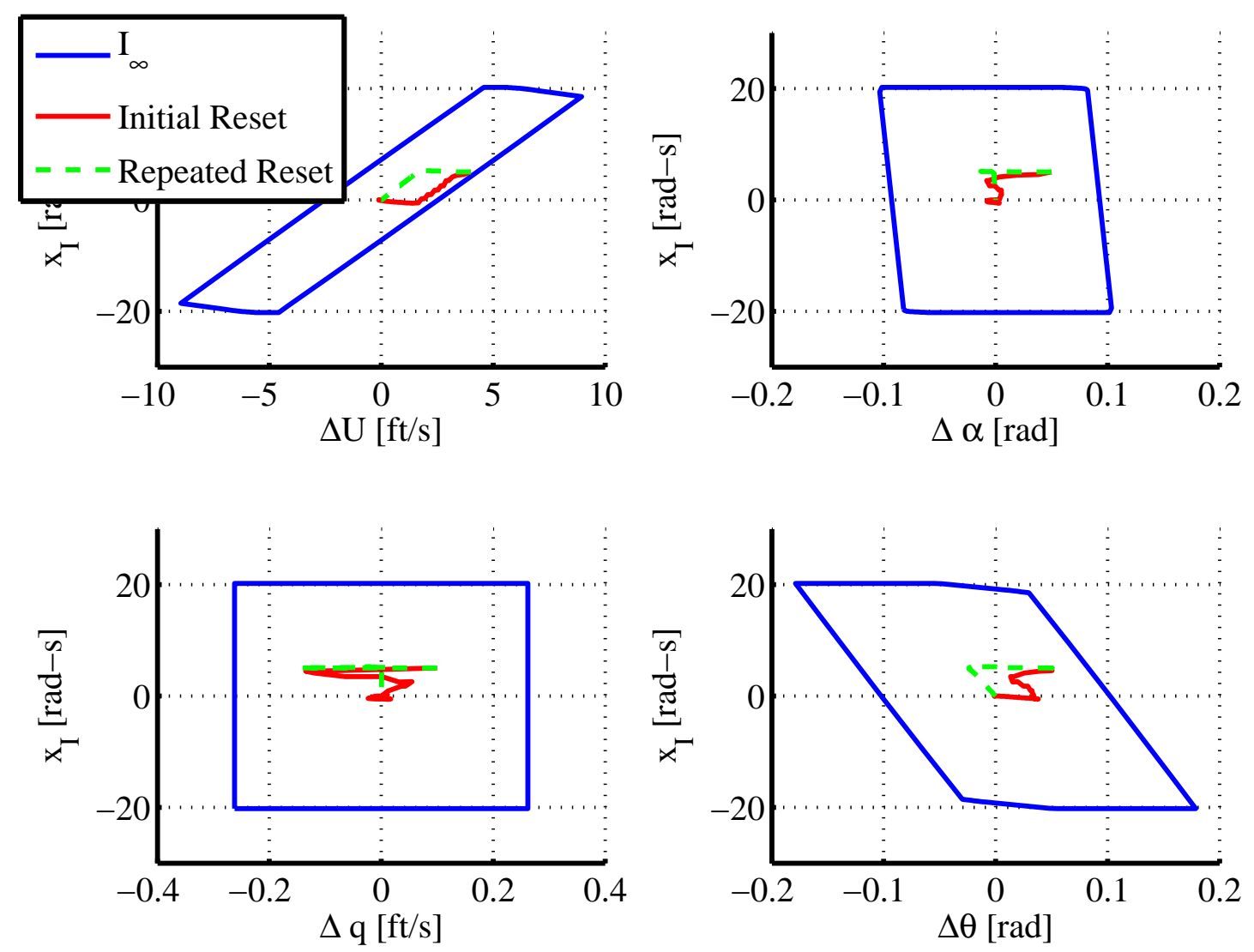

Figure 7: Projections of $I_{\infty}$ onto different planes and time histories of the longitudinal dynamics with an initial integrator state reset and with repeatedly resetting the integrator state. 

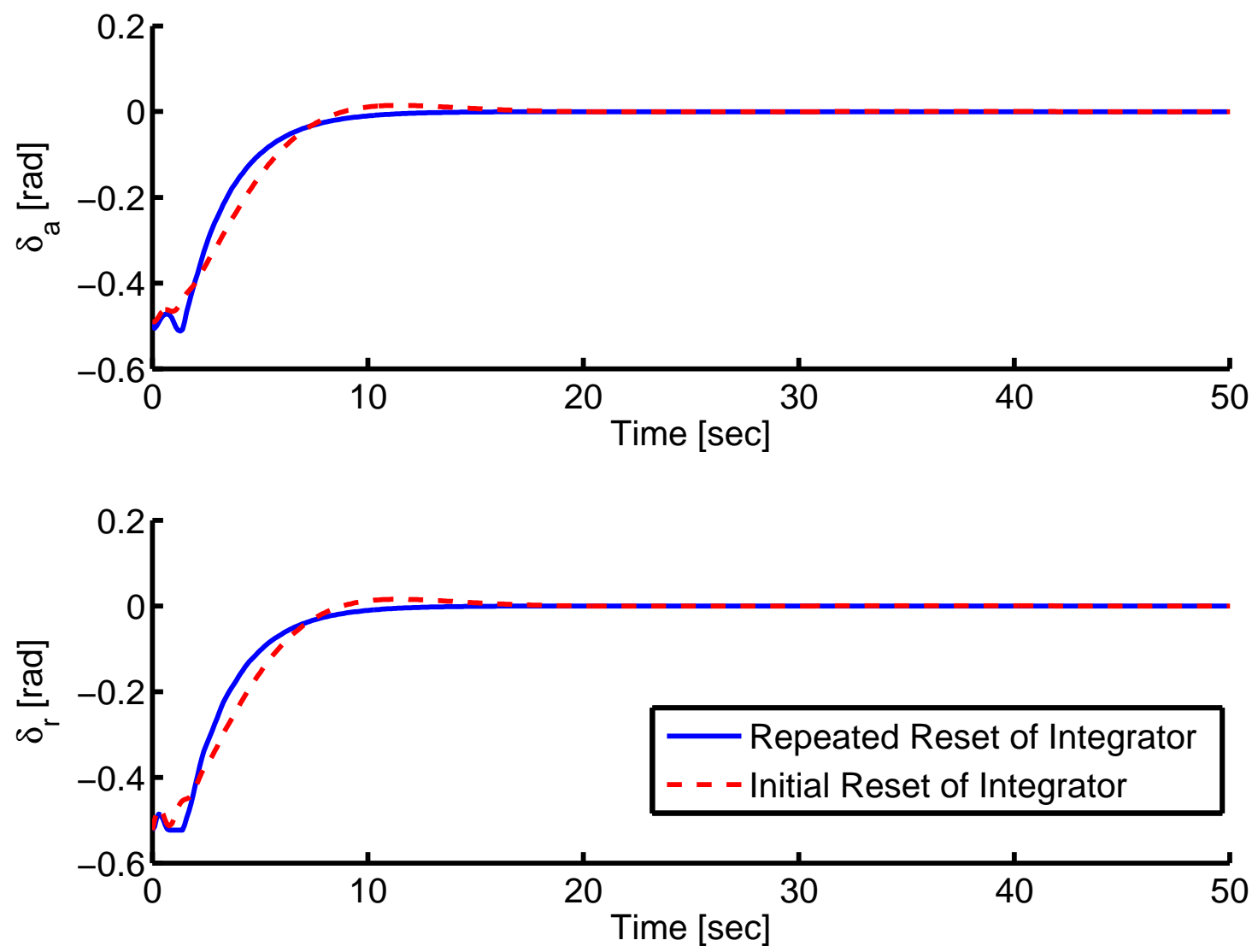

Figure 8: Comparison of the convergence of the lateral control inputs for an initial only integrator state reset versus repeated integrator state resets. 

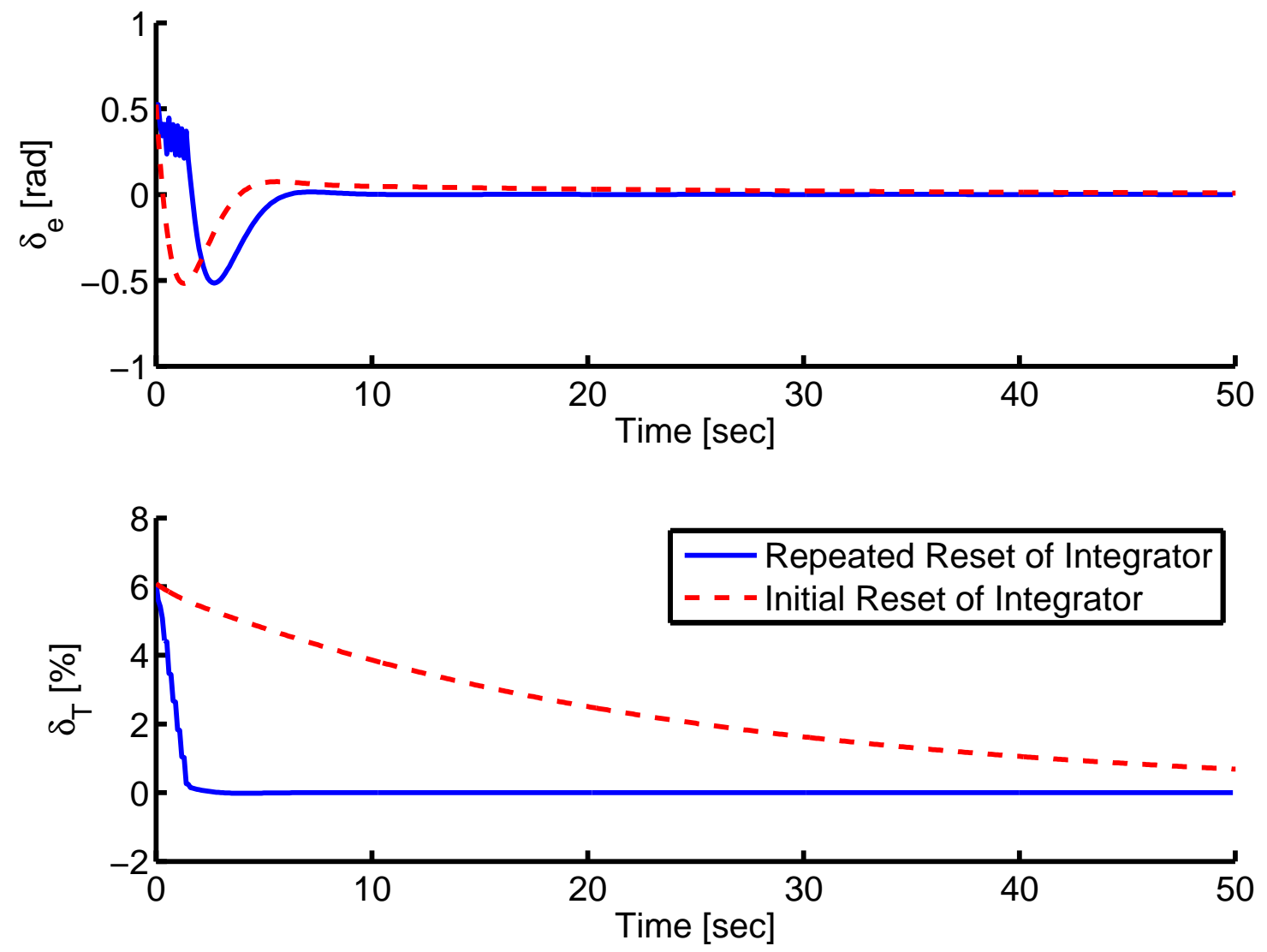

Figure 9: Comparison of the convergence of the longitudinal control inputs for an initial only integrator state reset versus repeated integrator state resets. Note the substantially quicker response with the repeatedly reset integrator state in the throttle case (bottom). 

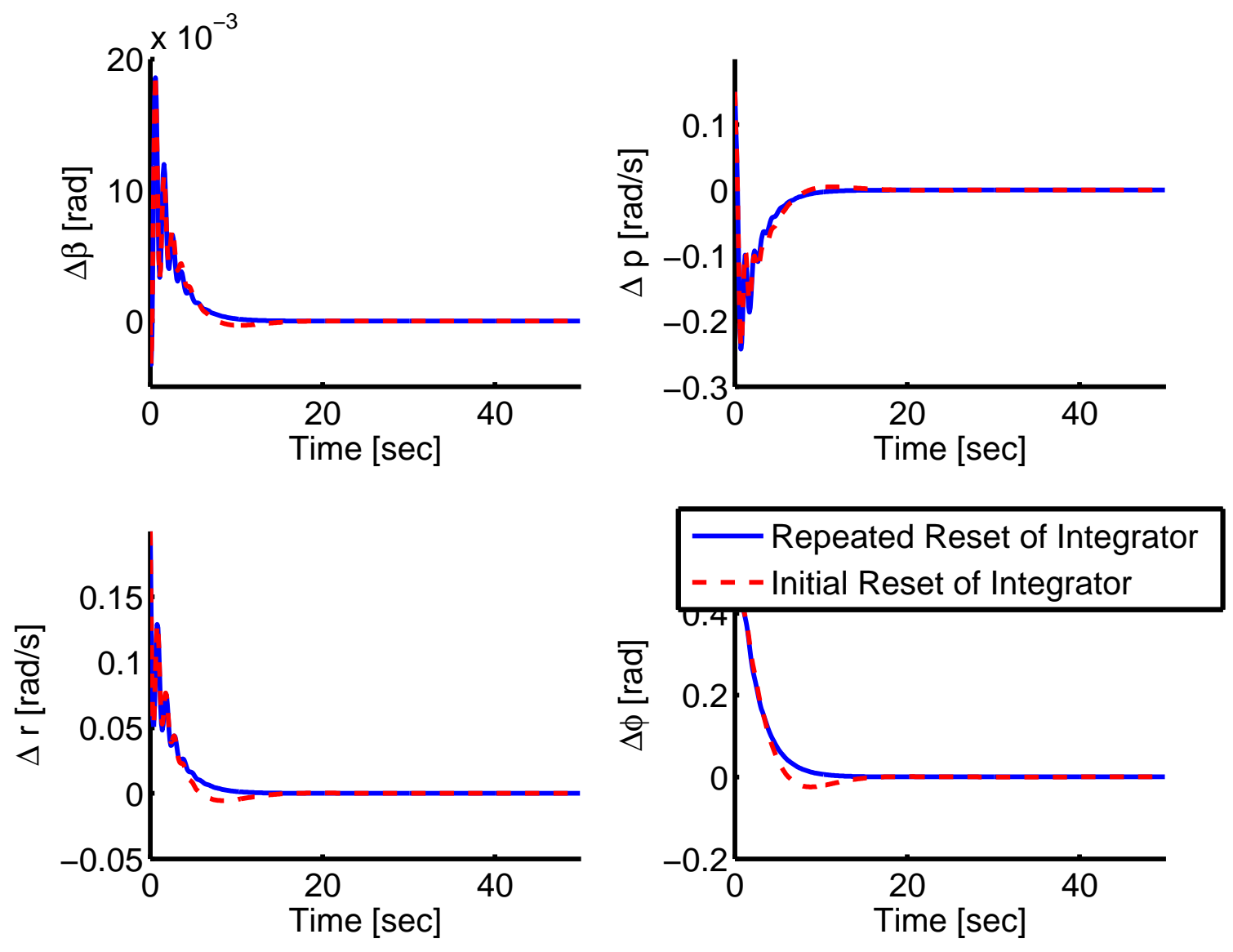

Figure 10: Comparison of the convergence of the lateral plant states for an initial only integrator state reset versus repeated integrator state resets. 

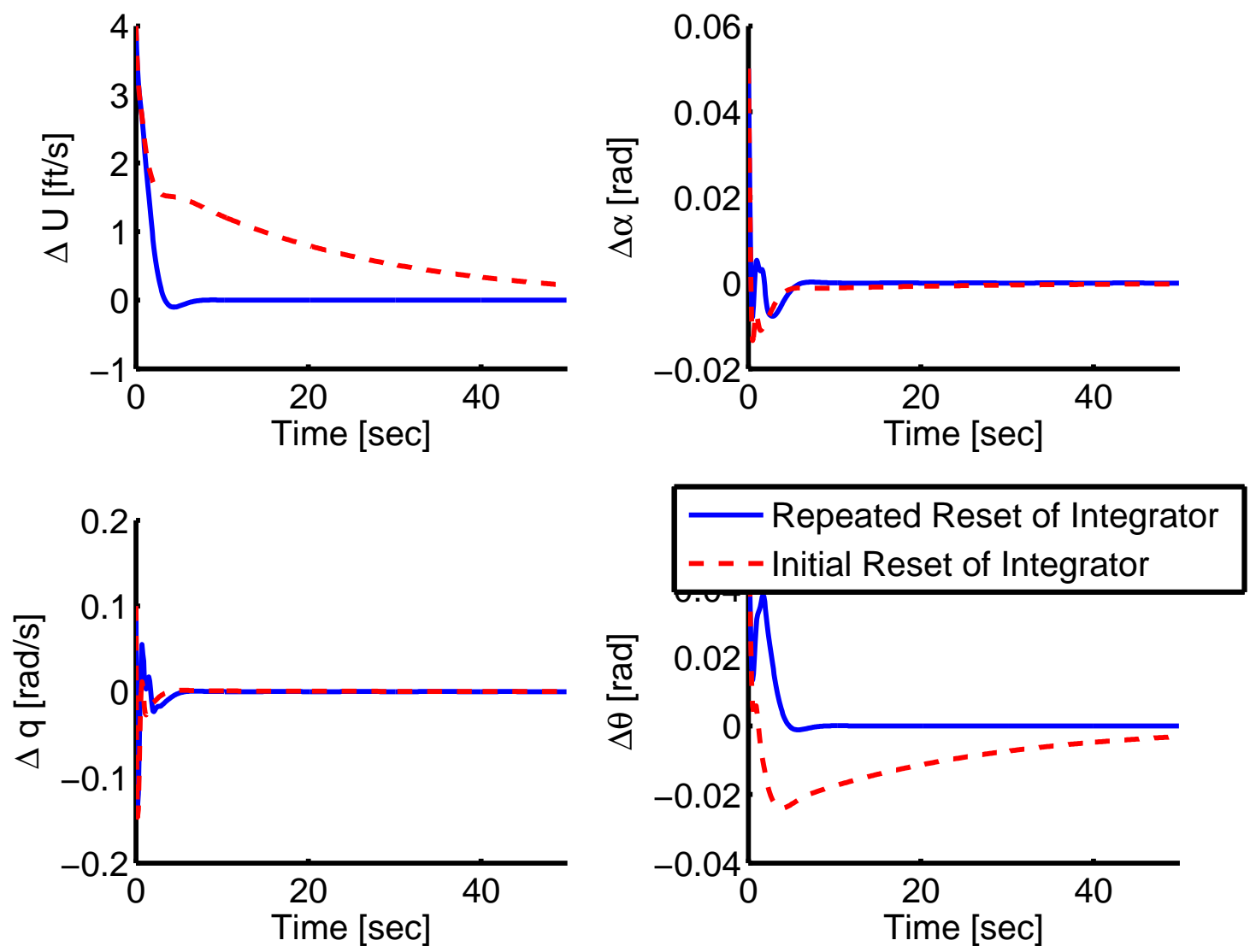

Figure 11: Comparison of the convergence of the longitudinal plant states for an initial only integrator state reset versus repeated integrator state resets. 

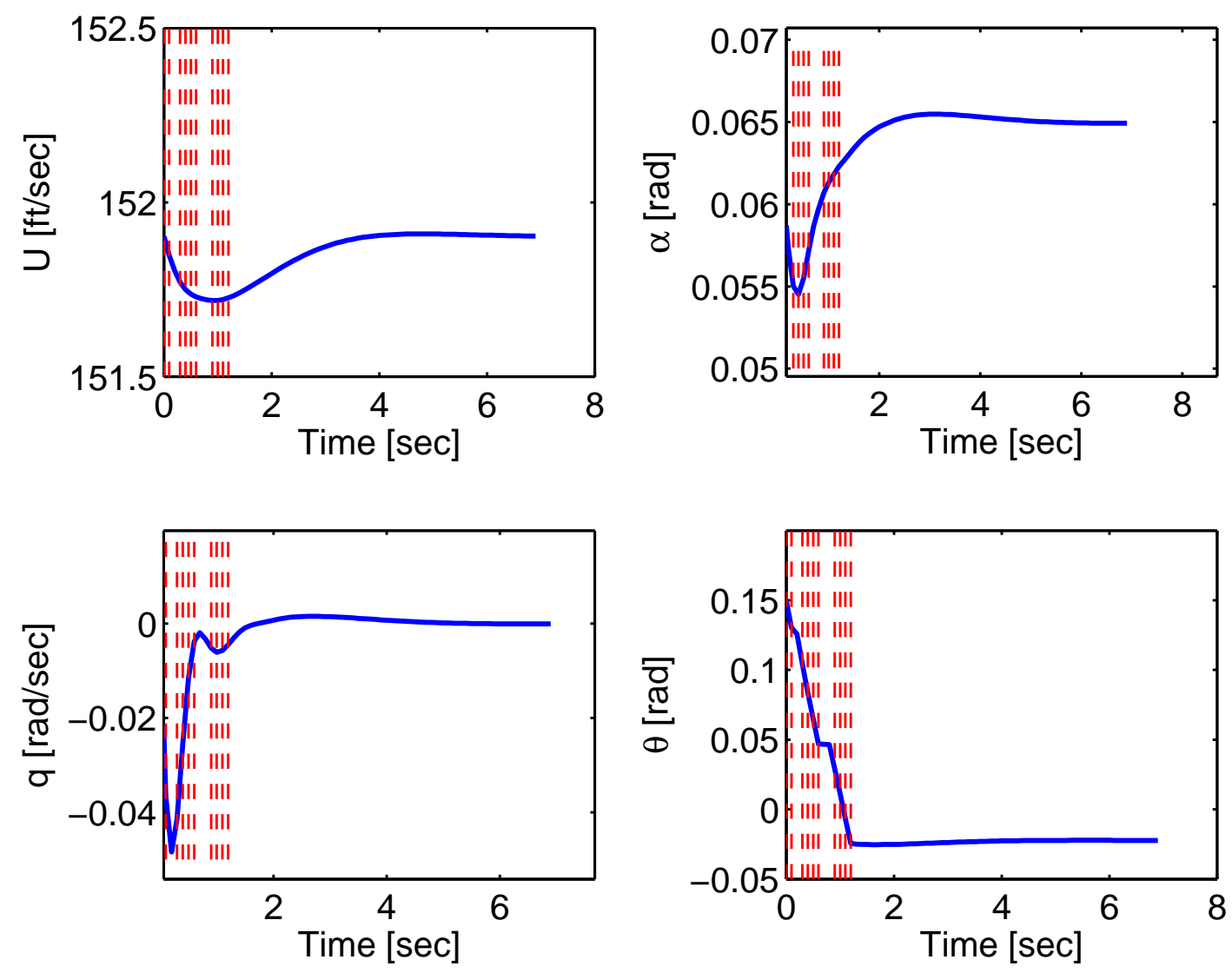

Figure 12: The time histories of aircraft states as the aircraft transitions through the selected trim points. The transitions between selected trim points occur at time instants designated by vertical dashed lines 

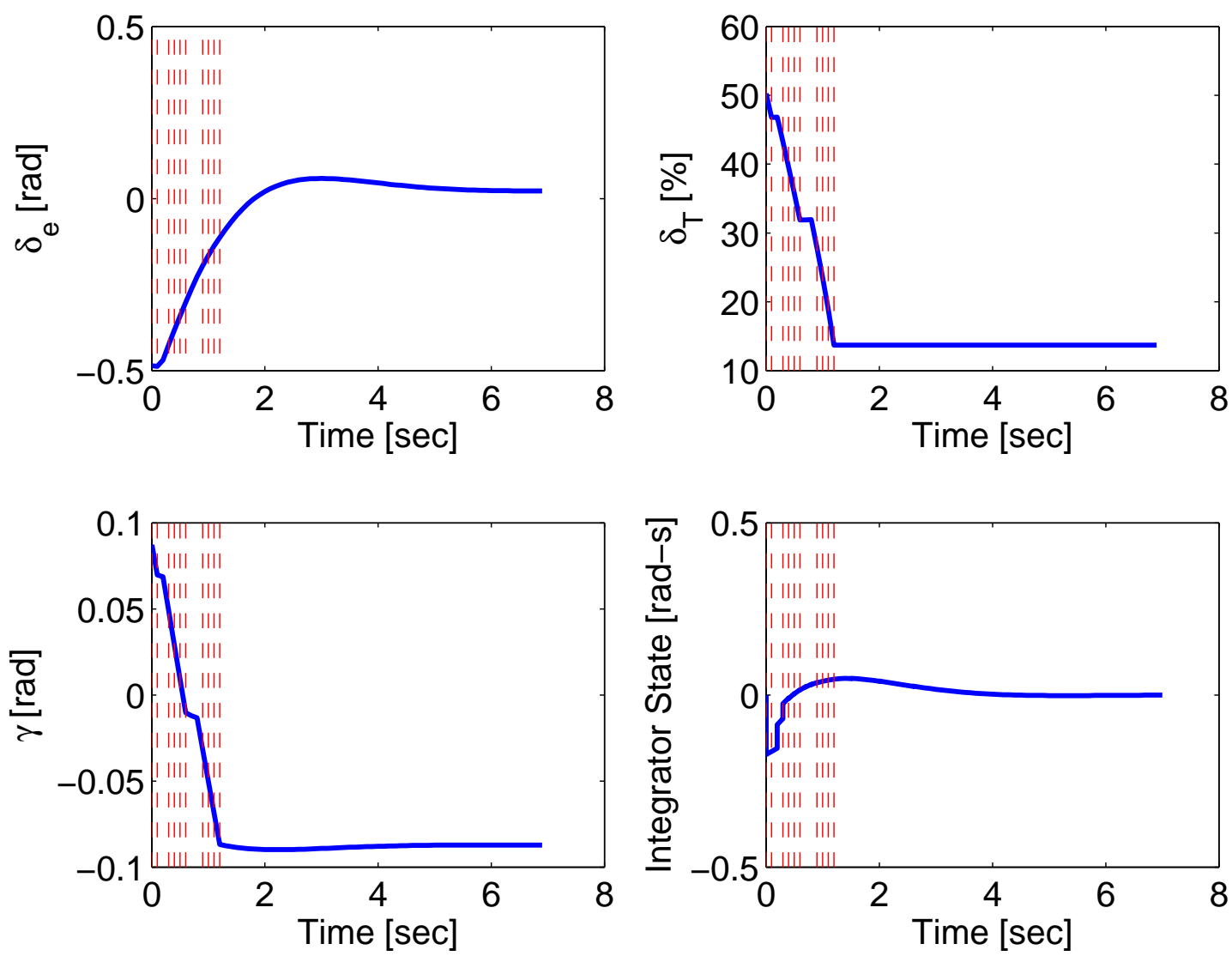

Figure 13: The control input, reference $\gamma$, and integrator state trajectories as the aircraft transitions through the selected trim points. Note the vertical jumps in the integrator state (bottom right) plot indicate the integrator state reset. The transitions between selected trim points occur at time instants designated by vertical dashed lines. 